\title{
Thoracoscopic Transdiaphragmatic Approach for Anterior Body Reconstruction of L2 Burst Fracture
}

\author{
Jong Tae Park \\ Department of Neurosurgery, Wonkwang University School of Medicine, Iksan, Korea
}

Corresponding Author:

Jong Tae Park, MD

Department of Neurosurgery,

School of Medicine, Wonkwang

University, 895, Muwang-ro, Iksan-si,

Jeollabuk-do 54538, Korea

Tel: +82-63-859-1460

Fax: +82-63-852-2606

E-mail: jtpark@wku.ac.kr

Received: May 4, 2016

Revised: July 18, 2016

Accepted: July 20, 2016
This study describes the surgical technique and results of video-assisted thoracic surgery (VATS) in a patient who underwent reconstruction of an unstable $L 2$ body fracture. There are various surgical treatment options for $L 2$ burst fractures. The optimal method remains controversial. We performed L2 body reconstruction using VATS. An 18-year-old female was seen in the emergency room with paraparesis grade 4 and was diagnosed with a burst fracture of L2. A two-stage procedure was planned. First, urgent posterior decompression and stabilization were performed with dorsal laminectomy and insertion of screw systems at L1 and L3 pedicles. Then, the L2 body was reconstructed with an expandable cage by VATS. Her weakness was improved immediately after decompressive surgery. This surgical approach is an alternative and minimally invasive method for the treatment of traumatic L2 burst fractures.

Key Words: VATS, L2 burst fractures, Expandable cage, Minimally invasive

\section{INTRODUCTION}

Video-assisted thoracoscopic spinal surgery (VATS) is a minimally invasive approach for access to the anterior pathology of the thoracic and thoracolumbar spine, and has evolved since the early 1990s, especially in the United States and Germany ${ }^{7,8}$. Various lesions around the thoracic spine are increasingly diagnosed and treated using thoracoscopy ${ }^{2,3,9)}$. VATS is currently indicated in a large number of processes and injuries. Thoracoscopy has been used in simple procedures such as biopsy and sympathectomy, and is now widely utilized for herniated discs, neoplastic or infectious lesions affecting the spine/disc space, corrective surgery for scoliosis, and removal and reconstruction of the vertebral body ${ }^{1,3,9)}$

Limitations of thoracoscopy include lack of familiarity with the surgical anatomy, surgical environment, and instrumentation, the need for skilled assistance, and the steep learning curve. Nonetheless, several surgeons have gained considerable experience $^{3,4)}$

Although reconstruction of the vertebral body using thoracoscopy is not widely used, especially in fractures of the thoracolumbar junction, considerable advances have been made and many surgical treatment results have been reported ${ }^{1,25,6)}$. The author has performed many thoracoscopic surgeries for fractures of the thoracolumbar junction, including the second lumbar vertebra (L2), and describes his technique and issues related to the surgery.

\section{Method of thoracoscopic surgery for L2 body reconstruction}

The patient is placed in a right lateral position with the legs bent and arms fixed. Sufficient PAD should be inserted and attached firmly under the right axilla in order to prevent damage to the brachial plexus and bedsore on knees. Utilizing C-arm fluoroscopy, proper alignment of the surgical site is verified. Then, the insertion sites of 4 ports are marked. A working channel, optical channel, traction channel, and suction channel are required. Proper and careful construction of these 4 channels is very important in thoracoscopic surgery. First, a $1-\mathrm{cm}$-long optical channel is constructed just above the L1 body. A working channel is then created by inserting a finger into the optical channel and pushing down on the diaphragm. The working channel must be parallel to the rib and about $2 \mathrm{~cm}$ long. Using the previous 2 channels, the traction and suction channels are constructed along the anterior axillary line. Surgery can be performed using these 4 channels. If the distance between the channels is too small, instrument conflict and discomfort in performing the surgery can occur. If the distance is too great, it is difficult to manipulate the longer instruments. Therefore, it is important to maintain adequate working space. 
Before removal of the L2 body, discectomies of L1-2 and L2-3 are performed. The L2 body is removed with chisels, curettes, and pituitary forceps under C-arm fluoroscopy or the endoscopic image. The remaining disc or soft tissue should be thoroughly removed, especially on the lateral edge or bottom. For anterior reconstruction, an expandable cage (Synex ${ }^{\mathrm{TM}}$, Synthes, USA) filled with cancellous autograft bone chips is placed in the vertebrectomy defect, gradually distracted, and locked in place. The morselized autograft from L2 and the bone graft substitute are inserted in and around the cage and then placed in the MACs-TL plate system (Aesculap, Germany) if necessary.

\section{CASE REPORT}

An 18-year-old female was seen in the emergency room because of back pain and paraparesis (grade 4) after a fall from the fifth floor. She had an unstable burst fracture of the L2 body. The vertebra was severely damaged; both pedicles were destroyed, with the fragments protruding into the spinal canal and pressing firmly against the nerves (Fig. 1).The posterior approach was adopted in this emergency, and decompression was performed using laminectomy while pushing the broken fragments forward toward the body and using rear screw insertion in L1 and L3. Three days later, we removed the destroyed $\mathrm{L} 2$ body with the thoracic endoscope, inserted the expandable cage, and reconstructed the body. We inserted the MACs-TL plate system and restored the torn diaphragm. Routine postoperative images confirmed that the reconstruction of the L2 body was successful and that lumbar lordosis was well-maintained (Fig. 2). The chest tube was removed in 5 days, and the patient left the hospital ambulatory 15 days after the operation.

\section{DISCUSSION}

Anterior surgery on a lumbar fracture is generally conducted using a retroperitoneal approach ${ }^{10)}$. Surgeons usually take the retroperitoneal approach for anterior surgery on $L 2$ fractures ${ }^{10)}$. However, based on extensive experience with thoracoscopic surgery on thoracolumbar fractures (T12 or L1), the author has also performed endoscopic surgery on L2 fractures. Although
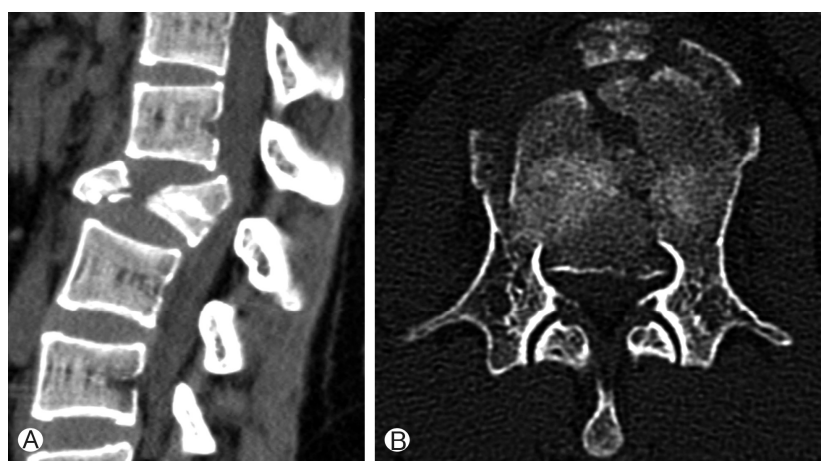

Fig. 1. Lumbar computed tomography shows a burst fracture of the L2 body. Sagittal image (A) axial image (B). it is known that thoracoscopy can reach to $L 3$, reconstructive spinal surgery on L2 fractures requires insertion of screws for the front plate into L3; therefore, reconstructive surgery of an L2 body fracture is probably the limit for thoracoscopic surgery. Sufficient experience with open surgery at the thoracolumbar junction is necessary. It is difficult to attempt endo-
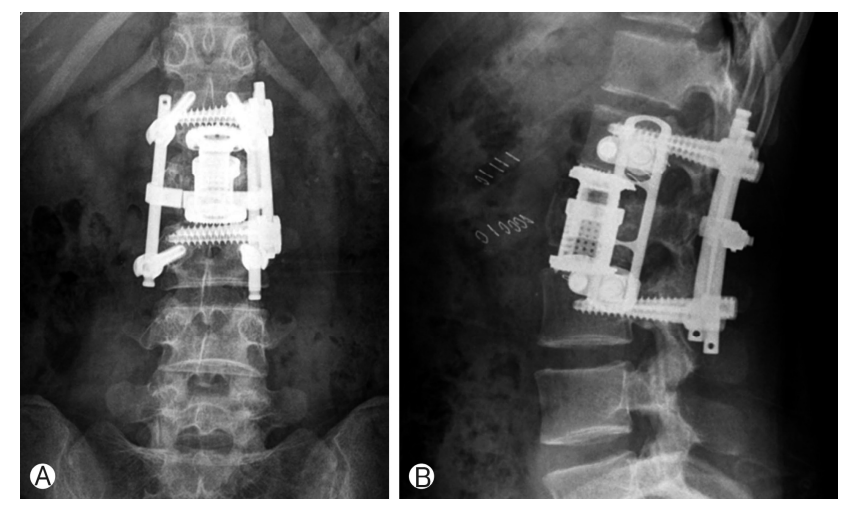

Fig. 2. Postoperative simple radiographs (A, B) show replacement of the L2 body with an expandable cage and MACs-TL plating system.

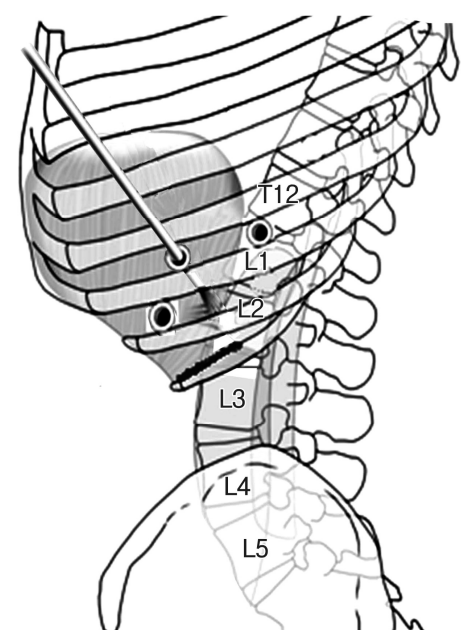

Fig. 3. Thoracoscopic transdiaphragmatic approach for L2 body can reach to L3 with resection and retraction of the diaphragm.

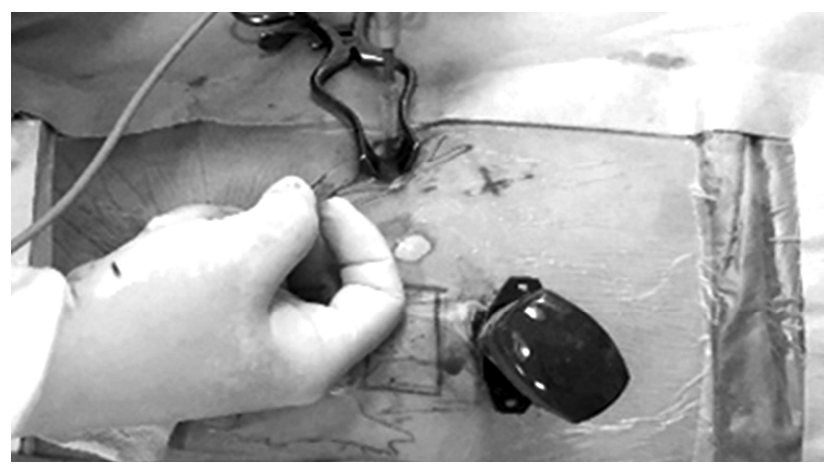

Fig. 4. This photograph shows finger dissection through the working channel to make a suction channel. 
scopic surgery on L2 without prior surgical experience on T12 and $L 1$, which require the least dissection of the diaphragm. A significant procedure in thoracoscopic surgery for thoracolumbar fracture is dissection of the diaphragm (transthoracic transdiaphragmatic approach) ${ }^{1,3-6)}$. L2 is entirely surrounded by the psoas muscle and lies under the diaphragm. Control of both the psoas muscle and the diaphragm is essential to be able to operate on L2 (Fig. 3). Thus, sufficient practice on T12 and L1 is recommended for the beginner. It is essential to make a long incision in the diaphragm and expose the thick psoas muscle up to L3 with sufficient dissection. The surgeon should also be experienced in restoring the diaphragm using an endoscope.

When an inexperienced surgeon inserts a port to make a working channel in the early stage of an operation, in most cases the port is near the retroperitoneal space instead of entering the thoracic cavity. To prevent this problem, the surgeon should first construct the optical channel, and then push downward with a finger to dissect the area where the diaphragm is attached to the rib (Fig. 4). At the same time, a working channel is constructed while externally touching the finger that entered through the optical channel. When the working channel is completed, the port does not enter the retroperitoneal space but can now access the thoracic cavity. Because a large incision is made, it is important to properly restore the diaphragm to avoid pneumonia or herniation of the stomach or large intestine.

\section{CONCLUSION}

We have performed reconstructive surgery using VATS in 11 cases of $L 2$ fracture in the past 10 years. This paper demonstrates that the operation is feasible by illustrating a series of successful surgical treatments, and describes the technique. More surgical results will enable reporting of more clinical and radiological features, as well as a comparison between thoracoscopic cases and cases treated with traditional open surgery.

\section{REFERENCES}

1. Beisse R. Endoscopic treatment of spinal trauma at the thoracolumbar junction. Indian J Orthop 41:277-85, 2007

2. Dickman CA, Rosenthal D, Karahalios DG, Paramore CG, Mican CA, Apostolides PJ, et al. Thoracic vertebrectomy and reconstruction using a microsurgical thoracoscopic approach. Neurosurgery 38:279-293, 1996

3. Huang TJ, Hsu RW, Liu HP, Hsu KY, Liao YS, Shih HN, et al. Video-assisted thoracoscopic treatment of spinal lesions in the thoracolumbar junction. Surg Endosc 11:1189-1193, 1997

4. Jung JR, Park SB Clinical and radiological analysis of Thoracoscopic Spinal surgery in thoracolumbar burst fracture. J Kor Neurotraumatol Soc 7:24-28, 2011

5. Khoo LT, Beisse R, Potulski M. Thoracoscopic-assisted treatment of thoracic and lumbar fractures: a series of 371 consecutive cases. Neurosurgery 51(5 Suppl):S104-17, 2002

6. Kim DH, Jahng TA, Balabhadra RS, Potulski M, Beisse R. Thoracoscopic transdiaphragmatic approach to thoracolumbar junction fractures. Spine J 4:317-28, 2004

7. Landreneau RJ, Mack MJ, Hazelrigg SR, Dowling RD, Acuff TE, Magee MJ, et al. Video-assisted thoracic surgery: basic technical concepts and intercostal approach strategies. Ann Thorac Surg 54:800-807, 1992

8. Mack MJ, Regan JJ, Bobechko WP, Acuff TE. Application of thoracoscopy for diseases of the spine. Ann Thorac Surg 56:736738, 1993

9. Niemeyer T, Freeman BJ, Grevitt MP, Webb JK. Anterior thoracoscopic surgery followed by posterior instrumentation and fusion in spinal deformity. Eur Spine J 9:499-504, 2000

10. Reinhold M, Knop C, Beisse R, Audigé L, Kandziora F, Pizanis A, et al. Operative treatment of 733 patients with acute thoracolumbar spinal injuries: comprehensive results from the second, prospective, Internet-based multicenter study of the Spine Study Group of the German Association of Trauma Surgery. Eur Spine J 19:1657-76. 2010 